\title{
The Hidden Abandonment
}

\author{
Irena Alimerko \\ PhD Candidate at European University of Tirana, Albania
}

\section{Doi:10.5901/jesr.2015.v5n1p35}

\begin{abstract}
A disturbing phenomenon that is happening nowadays in school is the hidden abandonment. There are a few children who leave schools, compared with children who attend the schools, but they are not in the care of their teachers in the classroom during the lessons. This phenomenon is displayed in the form of an apparent indifference from the pupils during the class as they come in the school unprepared. A part of them often disturb their coevals. The reasons of these acts are various. However, it is not important to point out only the reasons but, more than that, to know how to avoid this phenomenon. I say so, because the abandonment often is justified from the teachers as a phenomenon that occurs with the pupils that have come from the rural areas and have evident deficits in learning. The teacher must be careful in setting objectives on what knowledge should gain the pupils in the end of the class hour considering all the levels of them, as the best ones as those who have a lower average. The setting of minimal objectives takes an important role, so as the selection of methods, techniques and activities for the realization of these objectives, the premeditation of questions for these pupils and the specific tasks they have to make at home. Often it is noticed that these pupils come unprepared and the main reason is the lack of knowledge. Another important role in this process is the motivation of these pupils. Such motivations consist in: to draw the favorite personage, to memorize the poetry, etc. The most important thing is that the questions have to be such that require short answers and not annoying ones.
\end{abstract}

Keywords: hidden abandonment, the active role of pupils, educational development, motivation

\section{Introduction}

The learning process at school consists in joint activity of teachers and pupils and precisely through this joint activity the teacher transmits to the pupils the scientific knowledge. Considering the characteristics and the scientific requirements on the class organization, during which the teacher plays a leading role, we can observe the active role of pupils in learning in all important stages of class; the control of knowledge, the independent work, the creative projects, the strengthening of knowledge with the support of the teacher, the laboratory work, etc. The pupil perceives the knowledge given from his teacher and works independently with his support for gaining more knowledge, skills and habits. We must have discipline in order to have freedom; the freedom is developed through discipline. Thus the pupil is active when the teacher works with the whole class, so as with the whole, as well as with a particular individual pupil, with the purpose for giving the knowledge mostly in the necessary areas and topics, thanks to a bilateral collaboration.

A disturbing phenomenon that is happening nowadays in school is the hidden abandonment. There are a few children who leave schools, compared with children who attend the schools, but they are not in the care of their teachers in the classroom during the lessons. ${ }^{1}$ This phenomenon is displayed in the form of an apparent indifference from the pupils during the class as they come in the school unprepared. A part of them often disturb their coevals. The reasons of these acts are various. However, it is not important to point out only the reasons but, more than that, to know how to avoid this phenomenon. I say so, because the abandonment often is justified from the teachers as a phenomenon that occurs with the pupils that have come from the rural areas and have evident deficits in learning. These pupils have the inability to adapt themselves with the new geographical, psychological and social environment, with the new requirements that are higher than those that they were used to have in the area where they have come from.

\section{Overview}

We, as teachers don't always know our pupils. We come into the world as individuals, achieve character, and become

${ }^{1}$ Stavri Llambiri, The Hidden Abandonment, UNICEF, Tiranë, 2002, p.4. 
persons. ${ }^{2}$ The teachers must know the personality of each pupil.

What harms often our work on achieving the main objective, of providing the schooling obligation is linked with the lack of concentration on those groups of pupils that have problems of age and temper, which have specific psychological issues on the psycho-motoric and physic development, different from the majority... Such pupils are mostly prejudged. There are only a few teachers that know how to support and motive the positive attributes of these "difficult" "urchins" pupils. There are all treated the same. There is forgotten or is not recognized that the pupils are different in their psychocultural, social and educational development. Furthermore, this is more pronounced for the groups of pupils that are unfairly marginalized by life, by their parents, the community and schools.

Among these pupils is Henri .The boy has normal mental and physical development, as well as his coevals. He has had a positive run in school and good relations with the others, including the teachers, parents and the coevals. His family has had a little time of its permanent moving from the remote areas to the urban one. Time by time the school has become the opposite of what this place has to be as the second home; he didn't want any more to attend it. From the communication we had with him the reasons were related to his unability to be adapted with the new geographical, psychological and social environment as well as the new requirements that were higher compare to the area he has come from.

Spontaneously we wonder: is there something wrong with these pupils that are feeling bad in these circumstances? Do they have any responsibility for the differences in the assessment of previous schooling years and the actual one? The teachers "forget" that these arrival pupils go through a social, pedagogical, psychological and cultural crisis. They are neglected, differentiated from the others and not involved in the normal learning process. Such pupils are often prejudged, so as the teachers and the schools these pupils come from.

We have to admit that there is also a category of pupils who do not make systematic preparation that does not allow the appropriation of the acquired knowledge. Although the reasons that stimulate negatively this phenomenon are various, the purpose of this article is to bring out the solutions for avoiding the hidden abandonment. In order that each pupil won't feel abandoned during the class hour, the teacher must be careful in setting objectives on what knowledge should gain the pupils in the end of the class hour considering all the levels of them, as the best ones as those who have a lower average.

The setting of minimal objectives takes an important role, so as the selection of methods, techniques and activities for the realization of these objectives, the premeditation of questions for these pupils and the specific tasks they have to make at home. Often it is noticed that these pupils come unprepared and the main reason is the lack of knowledge. The teacher should aim to reach these pupils, motivating them to make their home duties charging the duties according the specific levels. For instance in the subject of language such pupils will be charged of copying the text of the poetry or the first exercises that are the easiest, appropriate for the level of these pupils. The most important is the concentration of the pupils in the learning process.

The teacher is one who makes developing two ideas, where there was only one. For this purpose the questions play an important role to make sure that the pupils are attending the class. These questions have to be appropriate for this category of pupils, according to their level of knowledge and will serve as a tool for them to remind and reproduce the lesson, for instance, to be able to say a definition, to draw the favorite personage, to memorize the poetry, etc. The most important thing is that the questions have to be such that require short answers and not annoying ones.

Another important role in this process is the motivation of these pupils. Such motivations consist in:

1- Feeling good in class. This may be achieved by giving them the right freedom to answer the questions even in the case of receiving the wrong answer. One of the effective techniques in this case is the "brain storm". This should be used for the whole class in order to avoid the underestimation toward the specific category of these pupils.

2- Stimulating the correct answers, not only by assessment, but also with expressions: -Very well! Go forward! I am satisfied! You have started to be well prepared, etc.

3- Encouraging by estimating them as well as giving the opportunities for them to make the self esteem. This may be achieved by organizing special topics in small groups where they will have space to express their thoughts.

It is noticed that the pupils who tend abandonment, often during the tests, they don't read at all the requirements and tend after 10 minutes to submit the paper. Or in other cases they tend to make copy from the others by disturbing them. In these cases the teacher has to draw the attention by saying: "Read the requirements and do what you

${ }^{2}$ Robert Ezra Park, Race and culture, Glencoe, III, "The Free Press", 1950, p. 250. 
know." That is why the teacher must include in the test the kind of questions that these pupils would have the opportunity to receive a satisfactory grade in the certain level. For making this happen, the teacher needs to make some previous minitests. In some cases, although the teacher may be careful on planning and implementing by respecting the above issues, still some pupils won't assimilate the concepts of their level.

\section{Methodology}

What can a teacher do, in order that these pupils won't remain in this level?!

His work should consist of:

1- Gathering information for knowing better the situation of the pupils, their weaknesses and gaps comparing to the baseline standards.

2- Establishing the objectives as well as a strategy for achieving these objectives.

In order to understand and give alternatives for the solution of the issues raised above we have chosen the method of observation with the hypothesis that the selection of methods, techniques and activities, such as the premeditation of questions made to pupils can give good result on working with this target group. Through this method we would like to verify and derive the necessary information as for our study, as well as the community of teachers. In this case we have taken a sample of a class with 30 pupils. The survey process was developed with the participation of the observer as well as through retention of data from direct observation. One of the important part of the process was the creating of a reliable report with the subjects of observation, in this case, with the pupils of the fifth grade in a secondary school. The observant has entered in relation with the participants becoming part of the group even through certain orientations and direct questions that would effect in the survey results.

I have chosen to concretize it with the subject of math of the fifth grade, by choosing the "Geometry". I chose this topic because I have noticed that most of pupils find difficulties with it. That's why I propose that the teacher organizes this class in the most interesting way he can. One of the techniques that will draw the attention of pupils is to make them designing the objects of this material.

During the consolidation stage (using the selected technique according to the structure) the teacher may detect the gaps of the pupils. For this will serve any test or control on their home duties. The results of the control will help the teacher to keep records in order to design appropriate exercises for the coming class, as well as the homework. The teachers can figure out other ways to detect deficiencies in order to support their completion. For this purpose I have planned to conduct an open hour with the topic: "A scheme for a set of rectangle".

The pupils were ordered to bring some geometric figures, mostly rectangle, as well as safety-match. A pupil's imagination had gone so far as he had built the geometric bodies with the help of yarns of match and plasticine. During the presentation of each work the others had to define the shown figures. Then they were split up into six groups and the leader of each group divided the tasks: each of them had to draw with a free hand or with the yarns of match a rectangular. I observed and made notes on how the pupils were able perceive by designing the rectangular.

This work in groups continued with another task; defining the attributes of rectangular from each pupil within the group. The leader of the group made notes on the board after the discussion in group. Then this work was shown to other groups who completed the whole scheme with the help of the teacher. I was keeping records on the level of knowledge for this category of pupils. The process of completion from other pupils could effect as a positive technique to remind the knowledge from the rest of pupils. Designing the figures in different ways could serve as enforcement of certain knowledge. At the end of this work the pupils were asked: -Do you like working together? The pupils were felt satisfied. Robert Ezra Park has written: "Everyone is always and everywhere, more or less consciously, playing a role. It is in these roles that we know each other; it is in these roles that we know ourselves". ${ }^{3}$ In this case the pupils can know each other as well as themselves.

\section{Results}

From my observations and the conversations with my colleagues about this topic, I have to highlight the fact that, if the teacher selects the creative forms as we have explained with the above with example, the phenomenon of the hidden abandonment will be reduced.

In order that each pupil won't feel abandoned during the class hour, the teacher must be careful in setting

${ }^{3}$ Robert Ezra Park, Race and culture, Glencoe, III, “The Free Press",1950, p. 250. 
objectives on what knowledge should gain the pupils in the end of the class hour considering all the levels of them, as the best ones as those who have a lower average. The setting of minimal objectives takes an important role, so as the selection of methods, techniques and activities for the realization of these objectives, the premeditation of questions for these pupils and the specific tasks they have to make at home.

The teacher is one who makes developing two ideas, where there was only one. For this purpose the questions play an important role to make sure that the pupils are attending the class. These questions have to be appropriate for this category of pupils, according to their level of knowledge and will serve as a tool for them to remind and reproduce the lesson, for instance, to be able to say a definition, to draw the favorite personage, to memorize the poetry, etc. The most important thing is that the questions have to be such that require short answers and not annoying ones.

Another important role in this process is the motivation of these pupils. Such motivations consist in: making them feeling good in class; stimulating them with the correct answers and encouraging by estimating them as well as giving the opportunities for them to make the self esteem.

If pupils will be observed not only in forms that we say, but also in others the teacher selects, the hidden dropout phenomenon will come disappearing. It is a behavior that makes all responsive and encourages us to look at least as a phenomenon that raises the rifle and that should be the focus of our social behavior and not at the end of "class".

\section{References}

Robert Ezra Park, Race and culture, Glencoe, III, "The Free Press",1950

Stavri Llambiri, The Hidden Abandonment, UNICEF, Tiranë, 2002 